\title{
EXPLOITATION OF MULTI-BAND LIDAR FOR THE CLASSIFICATION OF FREE-FLYING MIGRATORY BIRDS: A PILOT STUDY OVER ATHENS, GREECE
}

\author{
Samuel Jansson $^{1 *}$, Alexandros Papayannis ${ }^{2}$, Susanne Åkesson ${ }^{3}$, Georgios Tsaknakis ${ }^{2}$, Mikkel \\ Brydegaard $^{1}$ \\ ${ }^{1}$ Lund Laser Centre, Lund University, P.O. Box 118, SE-221 00 Lund, Sweden \\ ${ }^{2}$ Laser Remote Sensing Laboratory, National Technical University of Athens, 15780 Athens, Greece \\ ${ }^{3}$ Centre for Animal Movement Research, Lund University, Ecology Building, SE-223 62 Lund, Sweden \\ *Email: jansson.samuel@gmail.com
}

\begin{abstract}
A multi-wavelength lidar system was used to detect free-flying birds passing over Athens, Greece. The location is strategically located in one of the important migratory corridors for birds migrating between Europe and Africa. Multiwavelength aerosol lidars are operated regularly across Europe in the frame of EARLINET. Here, the feasibility of using this existing infrastructure for assessing fluxes of migratory birds is explored. The backscattered lidar signals were detected at three elastic bands and one Raman band. The monitoring was extended over a period of three months covering predominantly the summer and early autumn period during which approximately 100 hours of lidar data was gathered.
\end{abstract}

\section{INTRODUCTION AND BACKGROUND}

Bird migration is a global seasonal phenomenon, engaging billions of birds transporting themselves between distant locations used for breeding and wintering [1]. The long-distance migration has evolved repeatedly in birds and routes often follow topographical features that are concentrating in coastal areas before sea crossings [2]. Different species, age classes and populations depart at different times, and flight parameters such as timing, routes, and distances travelled differ substantially between them. It is therefore desirable to be able to identify migrants in flight. Furthermore, their movements can be affected by climate change [3], and as such the study of bird migration is of interest not only from a migration ecological point of view, but can also shed light on other large-scale phenomena.

Since a major part of the migration flights take place at night and at altitudes of several $\mathrm{km}$, bird migration is a challenging subject to study in detail. Whereas most of the atmospheric lidars pursue the monitoring of gases [4] and aerosols [5] our group has recently started exploring lidar monitoring of the atmospheric fauna, such as insects [6] and birds [7].

The EARLINET comprises 27 regularly operated lidar systems across Europe, each with at least three elastic bands, and a majority with several Raman channels [8]. Coincidentally, many of these systems are located inside or close to common migratory corridors of birds [1], and it is therefore of interest to investigate the potential of utilizing these existing lidar systems parasitically for the classification of migratory birds.

\section{SITE AND INSTRUMENTATION}

The present study is based on data acquired from the EOLE Raman lidar system [9], located at the National Technical University of Athens, Greece $\left(37.96^{\circ} \mathrm{N}, \quad 23.78^{\circ} \mathrm{E}, \quad 220 \mathrm{~m}\right.$ ASL). The measurements were performed between May 13 and July 25 in 2013, with a total of 100 hours of lidar data acquired during this period.

EOLE is based on a pulsed Nd:YAG laser with a $10 \mathrm{~Hz}$ repetition rate, emitting coaxially at 1064 , 532 and $355 \mathrm{~nm}$. The optical receiver is a $\varnothing 300$ $\mathrm{mm}$ modified Cassegrainian reflecting telescope, $\mathrm{f}$ $=600 \mathrm{~mm}$, coupled through an optical fiber and divided optically into six spectral bands. The laser beam and telescope field-of-view overlap entirely above $\sim 500 \mathrm{~m}$ above ground level. The elastic lidar bands at 532 and $355 \mathrm{~nm}$ and the $\mathrm{H}_{2} 0$ Raman band at $407 \mathrm{~nm}$ were recorded by photomultipliers, while the one at $1064 \mathrm{~nm}$ was recorded by an avalanche photodiode, with spectral bandwidths ranging between $0.4-1.5 \mathrm{~nm}$ FWHM.

The conventional LICEL GmbH electronics used with the system averaged 100-1000 shots for aerosol purposes (time resolution of $1.5 \mathrm{~min}$ ). Birds, however, would only appear in a single 
shot. Therefore a $5 \mathrm{GS} / \mathrm{s}$ 4-channel digital oscilloscope with $300 \mathrm{MHz}$ bandwidth (PicoScope 6400-series) was used parasitically on EOLE and used to sample the 355, 407, 532 and $1064 \mathrm{~nm}$ bands in single laser shot mode.

\section{DATA PROCESSING}

The data was recorded and stored in files, each file containing 4 bands with 20000 time-offlight/altitude bins, the first 1000 being pre-pulse and the remaining 19000 distributed over the lowest $4 \mathrm{~km}$ of the atmosphere, and 600 time slots, each file thereby spanning 1 minute in time. The occurrence of a bird being intercepted by a laser pulse can be considered scarce, and with close to 6000 files, each containing $48^{*} 10^{6} 16$-bit integer values, efficient algorithms for scanning through the data and extracting such bird events were needed. A false-color time-range map of a file containing a bird event is shown in Fig. 1.

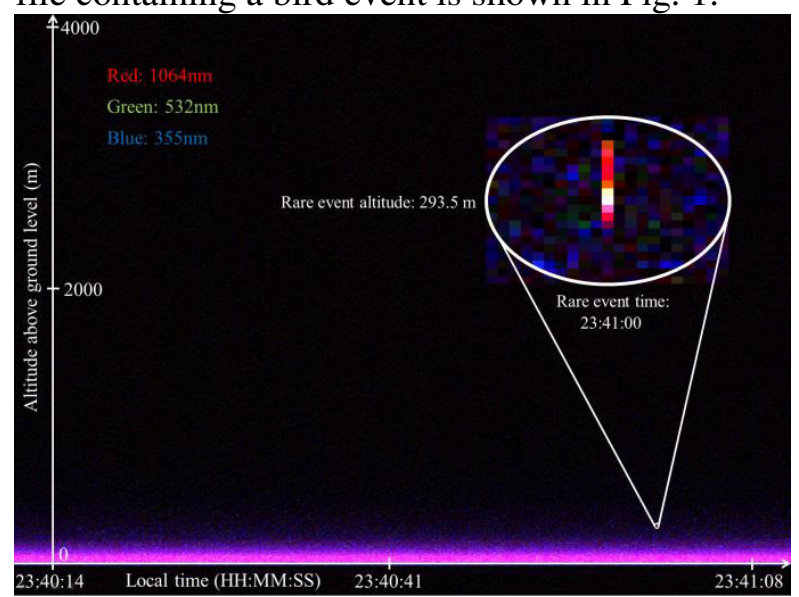

Figure 1: False-color representation of the three elastic bands of a data-file recorded at 27-05-2013 in Athens, Greece. The file contains a bird event at an altitude of 293.5 $\mathrm{m}$ above ground level at 23:41:00 LT.

To prevent false identifications, files recorded in inhomogeneous atmospheric conditions were excluded. This was done by fitting an analytical lidar form factor [10] against the static return signal in the $1064 \mathrm{~nm}$ channel. The files were rejected according to the residuals of the fit.

When a file was deemed to having been recorded in homogeneous atmospheric conditions it was scoured for bird events. First a detection threshold was set using the properties of the statistical intensity distribution in the elastic bands. In clean air the signal can be assumed to be normally distributed around a static value. The static return and the minimum signal were obtained, and the difference between the two was used to represent the noise amplitude. Events exceeding the static return plus twice the noise amplitude $(\mathrm{SNR}>2)$ were considered, and a threshold was set accordingly. The asymmetric intensity histograms for the three elastic bands, together with static return, minimum signal and threshold can be seen in Fig. 2. Lidar signals for the three elastic bands, together with the same three statistical parameters, are shown in Fig. 3.
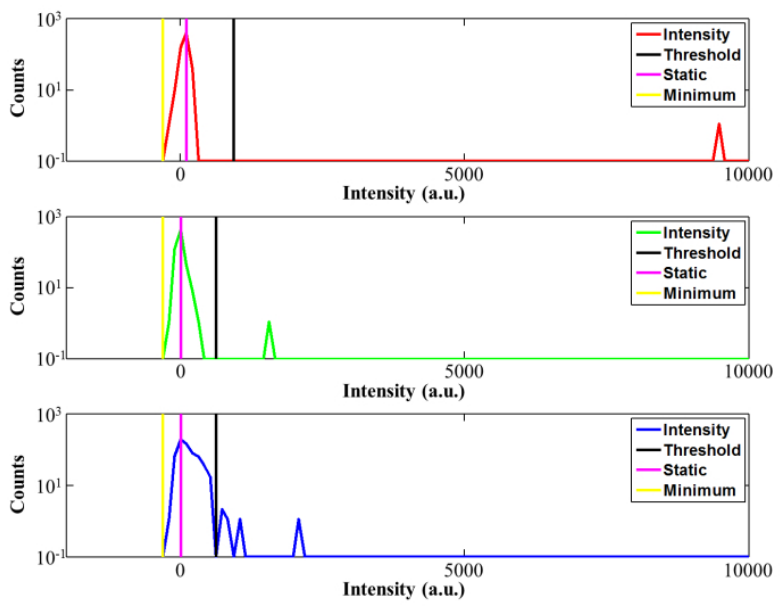

Figure 2: Intensity histograms for the three elastic bands at an altitude of $293.5 \mathrm{~m}$. The intensity distribution of the NIR, Green and UV bands is shown in the upper, middle and lower panels respectively. The static return, minimum signal and resulting threshold are shown in all three histograms. The event exceeds the threshold in all three bands.

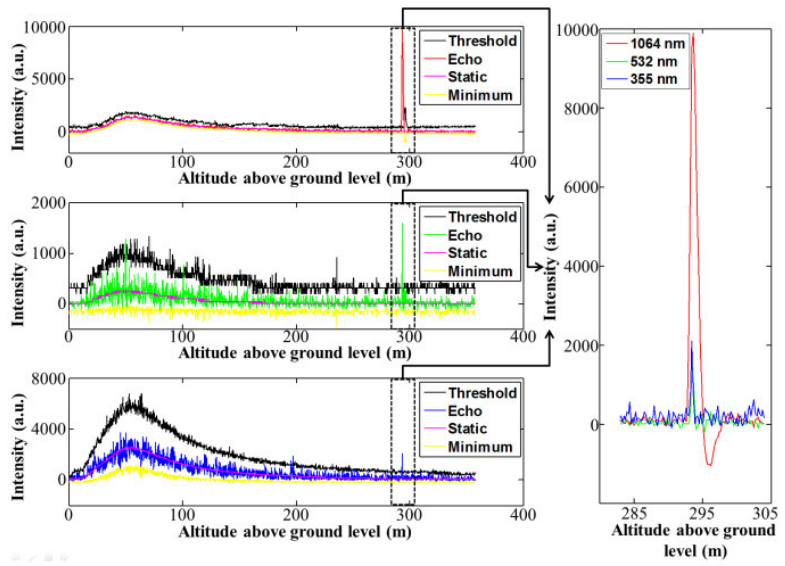

Figure 3: Lidar signals obtained at 23:41:00 LT using the same file as illustrated in Fig. 1. The upper, middle and lower panels show the intensities at 1064, 532 and $355 \mathrm{~nm}$ respectively, together with static return, minimum signal and threshold. The rightmost panel shows a close-up of the signals in the marked regions, exceeding the threshold in all bands simultaneously. 
While cosmic particles and single photons may cause spikes in the detectors, spikes exceeding the range dependent threshold in all three bands simultaneously were considered to arise from atmospheric fauna. Events fulfilling this criterion were considered as bird events and extracted for further analysis.

\section{PROPERTIES OF BIRD EVENTS}

Using the methods described in the previous section a total of 1735 events were found in homogeneous atmospheric conditions. The optical properties of these events were then further analyzed.

One of the properties analyzed was the lidar signal over the events in the $407 \mathrm{~nm} \mathrm{H}_{2} \mathrm{O}$ Raman band. Due to high atmospheric background this band was only active at night. Bird plumages consist of a keratin-air matrix with embedded melanin granules or other pigments [11] and can be expected to become fluorescent when excited by a $355 \mathrm{~nm}$ laser pulse [7]. The red-shifted light could then be detected in the $407 \mathrm{~nm}$ band. An example of the $407 \mathrm{~nm}$ lidar signal over a low altitude event can be seen in Fig. 4, together with the backscattered lidar signal at the three elastic bands.

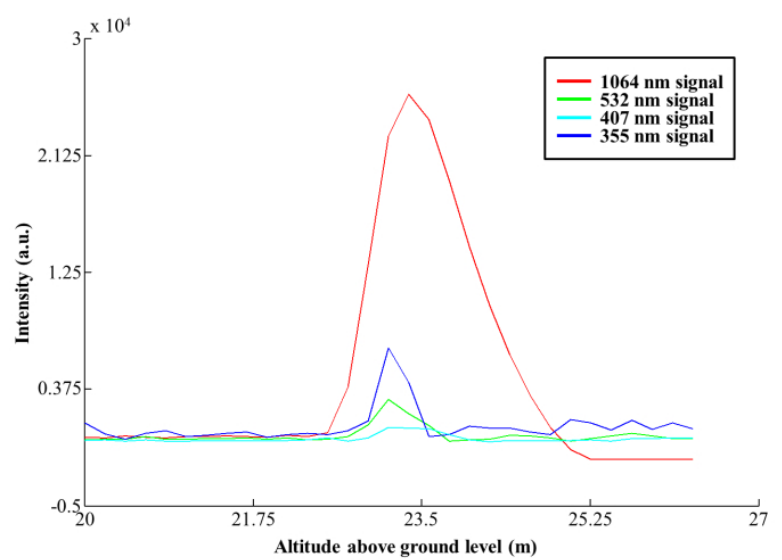

Figure 4: Signal in all four bands over a bird event.

Beam extinction was also analyzed, for the purpose of estimating bird-beam overlap. When part of the laser beam is blocked by an object the signal from the subsequent air volume will decrease, which could be used to assess the absolute reflectance of birds. An example of beam extinction after an event is shown in Fig. 5.

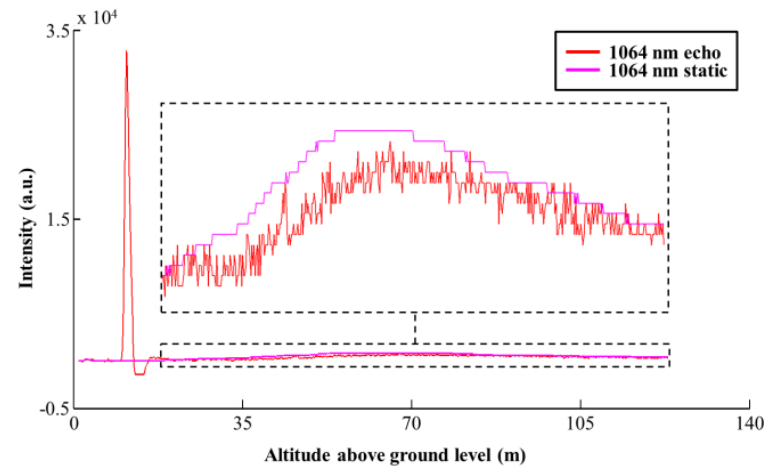

Figure 5: Laser beam extinction at $1064 \mathrm{~nm}$.

Beam extinction after events could, except for in a few cases, not be seen clearly. In the example above some $23 \%$ of the beam seems to have been blocked by the low altitude bird event and it is further observed that the signal recovers towards the static return at higher altitudes.

With an elastic backscatter signal in three bands, 3-point scatter spectra were constructed for all events. This was done by integrating the signal intensity over the event after subtracting and dividing with the static return, thus obtaining echo energies. These energies were then divided by the sum of all energies for the same bird event, thereby obtaining chromatic values. Fig. 6 shows a 2D histogram of the chromatic echo energies in the UV and Green bands.

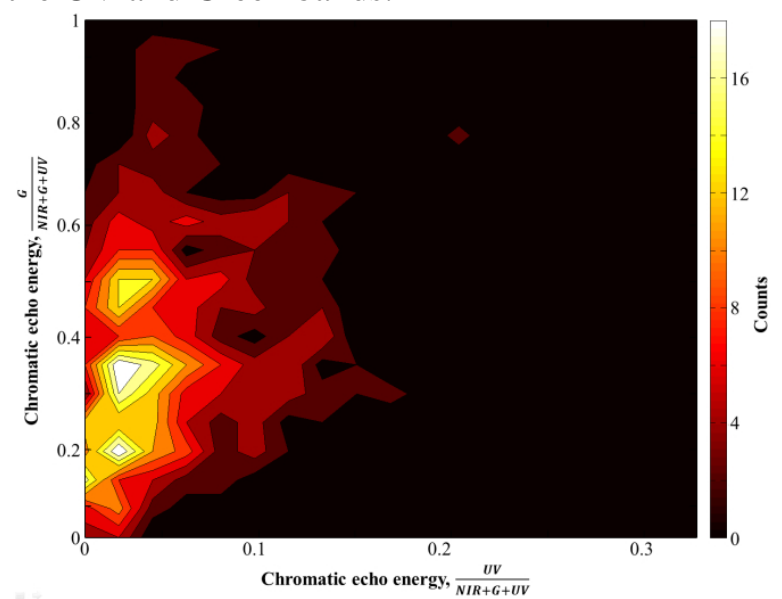

Figure 6: 2D histogram of the chromatic echo energies in the UV and Green bands. The congregation spots in this histogram could be associated with different observed bird species. 


\section{CONCLUSIONS}

A few aspects of the monitoring of free-flying birds using an aerosol lidar system have been illustrated in this study. The repetition rate of 10 $\mathrm{Hz}$ requires single shot observation and is somewhat inefficient for bird detection. Random noise spikes exceeding the threshold, specifically in the bands using photomultipliers could be circumvented by imposing that all bands spike simultaneously.

With fluorescence being a broadband phenomenon and the $407 \mathrm{~nm}$ band operating with a $0.41 \mathrm{~nm}$ filter bandwidth, any attempt to measure fluorescence induced in bird plumages can be assumed to be severely hampered. Though some spikes were observed at typical fluorescence timings they were too small to be significantly distinguished from noise. The inelastic band may be improved making it broader but would still only work at night.

The intent was to use beam extinction as a means of recalibrating the echo intensity into absolute reflectance, but since extinction could scarcely be detected this was not possible. It may, however, be possible at other concentric lidar nodes. In the presented example the bird event occurs before overlap between laser beam and telescope field of view is achieved, which is why the signal recovers at higher altitudes.

The peaks in the 2D histogram represent the most common reflectance spectra. These histogram regions with high event concentrations indicate a multimodality that could be related to different species of birds flying through the beam.

In this study it has been shown that it is feasible to monitor bird activity using the EARLINET and that in-flight species identification could be possible. Should wider filters be installed and the system run during migration seasons at night, many interesting studies could be performed. Furthermore, since the EARLINET encompasses 27 lidar systems, many of which are located in prime locations for the study of bird migration, this offers the opportunity of monitoring bird migration on a continental scale.

\section{ACKNOWLEDGEMENTS}

The study was financially supported by grants to S. A. from the Swedish Research Council (621-2013-4361) and Lund University. This project is a contribution from the Centre for Animal Movement Research financed by a Linnaeus grant from the Swedish Research Council (349-2007-8690) and Lund University. The financial support by the European Community through the ACTRIS Research Infrastructure Action under the $7^{\text {th }}$ Framework Programme under Grant Agreement no 262254 is gratefully acknowledged.

\section{REFERENCES}

[1] Newton, I., 2008: The Migration Ecology of Birds, Academic.

[2] Alerstam, T., Hedenström, A., and Åkesson, S., 2003: Long-distance migration: evolution and determinants, Oikos 103, 247-260.

[3] Cox, G., 2010: Bird migration and Global Change, Island Press.

[4] Papayannis, A., et al., 1990: Multiwavelength lidar for ozone measurements in the troposphere and the lower stratosphere, Appl. Opt., 29, 467476.

[5] Papayannis, A., et al., 2008: Systematic lidar observations of Saharan dust over Europe in the fram of EARLINET (2000-2002), J. Geophys. Res., 113, D10204, doi:10.1029/2007JD009028.

[6] Guan, Z., et al., 2010: Insect monitoring with fluorescence lidar techniques: field experiments, Appl. Opt., 49, 5133-5142.

[7] Lundin, P., et al., 2011: Remote nocturnal bird classification by spectroscopy in extended wavelength ranges, Appl. Opt., 50, 3396-3411.

[8] Pappalardo, G., et al., 2014: EARLINET: Towards an advanced sustainable European aerosol lidar network, Atmos. Meas. Tech., 7, 2389-2409.

[9] Kokkalis, P., et al., 2012: The EOLE lidar system of the National Technical University of Athens, Proc. 26 ${ }^{\text {th }}$ Intern. Laser Radar Conf., 629-632, Porto Heli, Greece, A. Papayannis and D. Balis and V. Amiridis (Eds), 25-29 June 2012.

[10] Kuze, H., et al., 1998: Field-of-view dependence of lidar signals by use of Newtonian and Cassegrainian telescopes, Appl. Opt., 37, , 31283132.

[11] Hill, G. E., and McGraw, K. J., 2006: Bird coloration vol. 1., Mechanisms and measurements, Cambridge, Massachusetts: Harvard University Press. 